\title{
Minimally invasive surgery for uterine fibroids
}

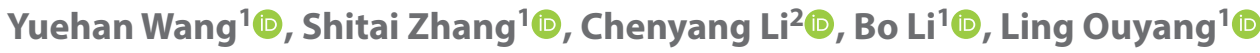 \\ ${ }^{1}$ Department of Obstetrics and Gynecology, Shengjing Hospital of China Medical University, Shenyang, China \\ ${ }^{2}$ Shenyang Maternity and Child Health Hospital, Shenyang, China
}

\begin{abstract}
The incidence of uterine fibroids, which comprise one of the most common female pelvic tumors, is almost 70-75\% for women of reproductive age. With the development of surgical techniques and skills, more individuals prefer minimally invasive methods to treat uterine fibroids. There is no doubt that minimally invasive surgery has broad use for uterine fibroids. Since laparoscopic myomectomy was first performed in 1979, more methods have been used for uterine fibroids, such as laparoscopic hysterectomy, laparoscopic radiofrequency volumetric thermal ablation, and uterine artery embolization, and each has many variations. In this review, we compared these methods of minimally invasive surgery for uterine fibroids, analyzed their benefits and drawbacks, and discussed their future development.
\end{abstract}

Key words: minimally invasive surgery; uterine fibroid; laparoscopic hysterectomy; laparoscopic myomectomy

Ginekologia Polska 2020; 91, 3: 149-157

\section{INTRODUCTION}

Uterine fibroids comprise one of the most common female pelvic tumors. When including the small, clinically undetectable fibroids and microscopic fibroids, the incidence is approximately $70-75 \%$ for those of reproductive age. The cause of uterine fibroids is not clear, and most fibroids present with no symptoms. Only 20-50\% fibroids have obvious symptoms, submucous type particularly, for example, abnormal uterine bleeding, urinary frequency or retention, obvious abdominal or pelvic pressure, and infertility. Most fibroids do not need treatment. However, indications for therapy include anemia resulting from metrorrhagia, pelvic pain or pressure affecting daily life, uterine compression, rapid tumor growth, tumor growth after menopause, and infertility [1].

The first laparoscopic myomectomy was performed by Semm in 1979 [2], and it may be the first minimally invasive surgery recorded. Since then, there have been numerous advancements in minimally invasive surgery for uterine fibroids. With the increasing of number of surgical methods and development of surgical techniques, uterine fibroid surgery is becoming easier, more feasible, and less invasive and results in fewer complications. Minimally invasive surgery has been considered an advanced approach for dealing with uterine fibroids. In 2014, Chittawar et al performed a meta-analysis to compare minimally invasive surgical techniques and open myomectomy for uterine fibroids. They found that those two kinds of surgery did not have different recurrence risks, but that laparoscopic myomectomy may be associated with less postoperative pain, lower postoperative fever, and shorter hospital stays compared with all other types of open myomectomy [3]. Minimally invasive surgery truly has its own advantages when dealing with uterine fibroids.

In this review, we compared the techniques, methods, and complications of many types of minimally invasive surgery to analyze their indications, advantages, and disadvantages (Tab. 1). We also evaluated their development status and have provided some evidence of the future development of minimally invasive surgery for uterine fibroids.

\section{Laparoscopic hysterectomy}

In 1989, Harry Reich performed the first laparoscopic hysterectomy [4]. Laparoscopic hysterectomy has developed into many types, with three of most common being total laparoscopic hysterectomy (TLH), laparoscopic-assisted vaginal hysterectomy (LAVH), and laparoscopic supracervical hysterectomy (LASH).

\section{Total laparoscopic hysterectomy (TLH)}

The TLH procedure has some features of laparoscopic surgery and abdominal hysterectomy. The use of the trocar is comparable to that of conventional laparoscopic myomec- 


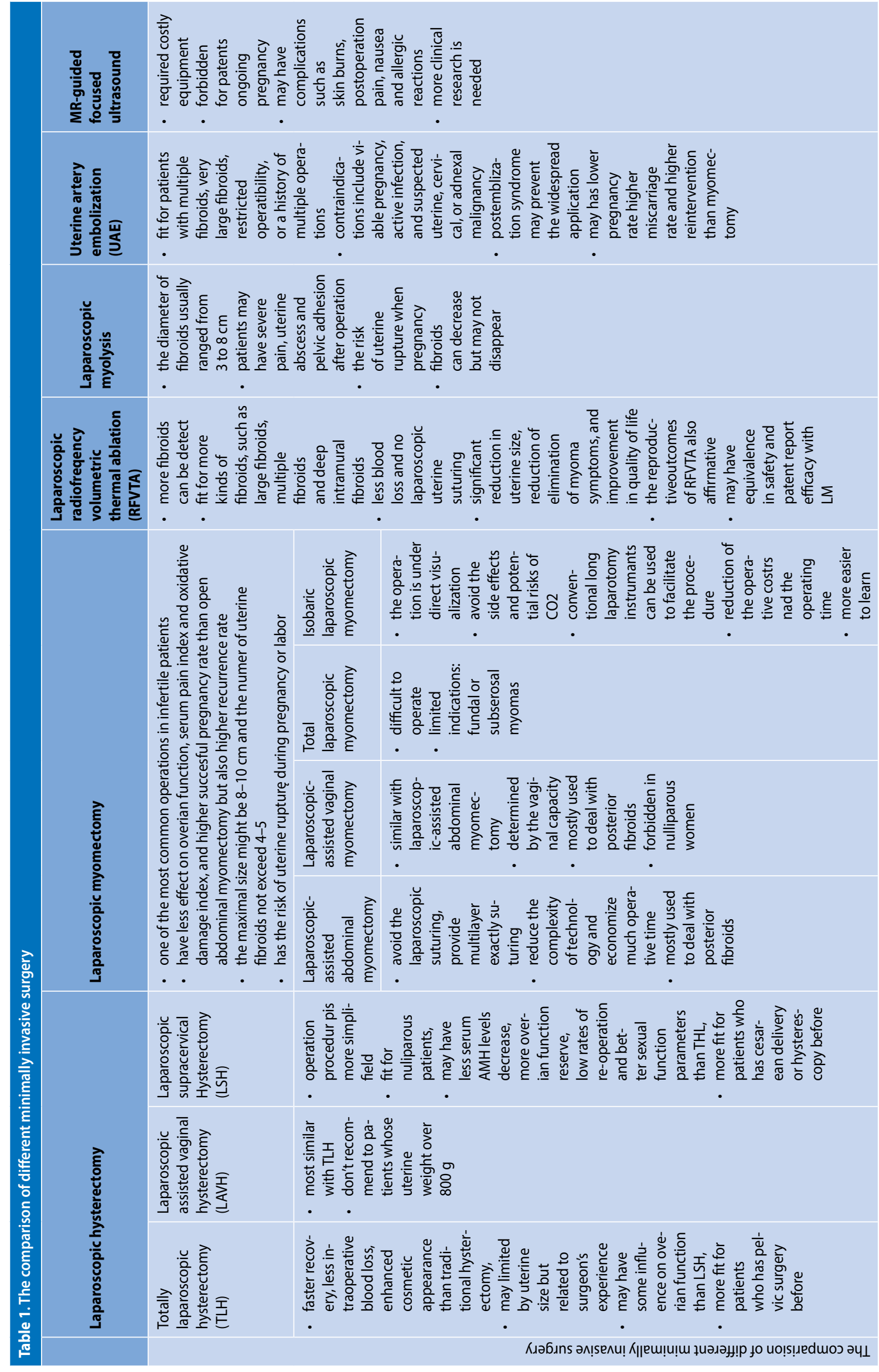


tomy. Pneumoperitoneum with $10-14 \mathrm{mmHg}$ needs to be created and three trocars are usually needed. The advantages of TLH compared with traditional abdominal hysterectomy include faster recovery, less intraoperative blood loss, less postoperative pain, and enhanced cosmetic appearance. Surgeons have discussed the largest fibroid weight that is still treatable with TLH. In 2017, Antonio et al reported TLH for a uterus containing $5352 \mathrm{~g}$ of fibroids [5]. Therefore, uterine size may no longer be a factor that influences whether to use laparoscopic hysterectomy. However, according to a multivariable analysis, there are some factors that present a high risk for conversion to open surgery, such as the surgeon's experience and fibroids with a maximum diameter $>10 \mathrm{~cm}$ [6].

\section{Laparoscopic-assisted vaginal hysterectomy (LAVH)}

Most LAVH procedures are akin to TLH, except for the vaginal procedure. The vaginal procedure begins after coagulating and resecting the ovarian ducts and proper ovarian ligaments. Surgeons should first dissect the bladder from the surface of the uterine and then open the Douglas pouch. Then, surgeons resect and ligate the cardinal ligament and enter the vesicouterine pouch. The next step is to ligate and resect the uterine arteries so that the uterus can be removed. Finally, hemostasis and suturing of the vaginal walls are performed before proceeding with the normal laparoscopic hysterectomy [7]. The greatest merits of LAVH compared with conventional abdominal hysterectomy are its reduced morbidity and faster recovery. The main factor limiting the use of LAVH is the uterine size. Although the maximum uterine size for $\mathrm{LAVH}$ is based on the experience and proficiency of the surgeon, when the uterine weight is more than $800 \mathrm{~g}$, LAVH may not be appropriate because of the significant blood loss or other complications. However, LAVH may be safe for patients with a uterine size $\leq 12 \mathrm{~cm}$ [7].

\section{Laparoscopic supracervical hysterectomy (LSH)}

The LSH procedure is contrary to that of TLH except for the preservation of the cervical stump and vaginal and uterosacral ligaments; therefore, the operative procedure is obviously simplified and the possibility of accidental injury to the surrounding organs such as the bladder, intestine, and ureter is reduced. Its indications are wider than those for LAVH; they include pain and/or uterine enlargement caused by myomata, dysfunctional uterine bleeding with no response to treatment, suspected uterine adenomyosis, and bleeding after endometrial ablation or resection. LASH is also suitable for nulliparous patients who have not experienced a vaginal delivery. It is a new minimally invasive alternative with low preoperative morbidity for total hysterectomy with benign conditions. Furthermore, LASH has benefits such as shorter hospital stays, faster recovery, and faster return to the workplace [8].
Research has been performed to determine the differences between TLH, LAVH, and LSH. LSH may involve lower serum AMH levels, more ovarian function reserve, low rates of re-operation and spotting, and better quality of life and sexual function than TLH [9-11]. Previous gynecologic conditions were also associated with the type of laparoscopic hysterectomy (LH) performed. Patients with a previous cesarean delivery and previous hysteroscopy are more likely to undergo LSH than LAVH. However, TLH is more suitable than LSH for patients who have undergone previous pelvic surgery. Estimated blood loss, operative time, and length of hospital stay were significantly reduced with LSH. Furthermore, LSH was the most common approach and was associated with significantly less morbidity [12].

\section{Laparoscopic myomectomy}

Laparoscopic myomectomy was first described by Semm in 1979; at that time, it was only used for subserous fibroids. In the early 1990s, this procedure was also used for intramural fibroids [13]. Currently, laparoscopic myomectomy is a common procedure for infertile patients. It is indicated for many conditions such as the presence of subserous or intramural fibroids that narrow the uterine cavity, myomas (which can be larger than $3 \mathrm{~cm}$ ), or multiple fibroids. Compared with traditional open myomectomy, laparoscopic myomectomy may have less of an effect on ovarian function, the serum pain index, and the oxidative damage index, and it may result in higher successful pregnancy rate. However, the recurrence rate with laparoscopic myomectomy might also be higher $[14,15]$. The general opinion is that the fibroid must have gradually emerged and have a maximal size of $8-10 \mathrm{~cm}$, and the total number of uterine fibroids should not exceed four or five [16]. Laparoscopic myomectomy is also associated with complications such as uterine rupture during pregnancy or labor, embolism, thrombosis, bowel injury, ureter injury, urinary bladder injury, excessive bleeding, and fistula.

With the constant development of techniques and instruments, the range of application for laparoscopic myomectomy is becoming wider, the procedure is becoming more intricate, and the complications are decreasing. Laparoscopic myomectomy has gradually improved; it results in reduced pain, shorter recovery time, and less ileus time than conventional laparotomy.

There are four different laparoscopic myomectomy approaches: laparoscopic-assisted abdominal myomectomy (LAAM); laparoscopic-assisted vaginal myomectomy (LAVM); total laparoscopic myomectomy (TLM); and isobaric laparoscopic myomectomy.

Laparoscopic-assisted abdominal myomectomy (LAAM)

LAAM was first performed by Nezhat et al. [17] in 1994. There is some concern that laparoscopy cannot be used 
to approach the exact wound site and realize the same hemostasis as conventional laparotomy; therefore, hematoma might occur after surgery. With LAAM, the fibroids are isolated, followed by a Mini-laparotomy. Therefore, laparoscopic suturing can be avoided. Compared with conventional laparoscopic myomectomy, LAAM is superior because it provides exact multilayer suturing, requires less complex technology, reduces the operative time, and results in similar recovery time. Furthermore, during laparoscopy, the general use of electrocoagulation hemostasis may injure the uterine tissues.

\section{Laparoscopic-assisted vaginal myomectomy}

During LAVM, a guide suture is usually placed to identify the largest tumor after laparoscopic identification of the location of all fibroids. Then, a culdotomy incision was made, through which the guide suture can be taken out of the uterus and placed in the vagina by using a grasper. These procedures usually involve enucleation and removal of the fibroids or repair of the uterine injury and hemostasis [1].

The benefits of LAVM are comparable to those of LAAM. However, there are some differences in the location of the fibroids. Generally, LAAM is used to manage fibroids developing in the anterior uterine or pedunculated fibroids, whereas LAVM is more suitable for posterior fibroids. The difficulty of LAVM is determined by the vaginal capacity. Therefore, LAVM is not recommended for nulliparous women and those with a contracted pelvis.

\section{Total laparoscopic myomectomy}

TLM is still considered a difficult laparoscopic procedure because repairing the uterine defect can be challenging. TLM has limited indications, such as fundal or subserosal myomas. Laparoscopic enucleation of large or deep intramural fibroids remains debatable. To solve this problem, Yuen et al. [18] proposed an improved laparoscopic suturing technique that involves the surgeon manually controlling the tail of the suture while sewing laparoscopically. However, this method may be of little value for the skilled surgeons.

\section{Isobaric laparoscopic myomectomy}

Most laparoscopic myomectomy procedures use $\mathrm{CO}_{2}$ to build pneumoperitoneum; however, when the myoma is large ( $\geq 8 \mathrm{~cm})$, surgery may be hampered because of the increased operative time, risk of preoperative bleeding, and risk of conversion to laparotomy [19]. Hence, a new method called isobaric laparoscopic myomectomy was developed. With this method, surgeons use a laprotenser to lift the abdominal wall so that a vertical intraumbilical incision can be made. Then, primary access is realized by inserting a $10-\mathrm{mm}$ to $11-\mathrm{mm}$ trocar through that incision, and two lower incisions are made without trocars lateral to the rectus muscles. The incision on the right side is $15 \mathrm{~mm}$ to $20 \mathrm{~mm}$, and that on the left side is $10 \mathrm{~mm}$ [19]. Therefore, under direct visualization, conventional long laparoscopic instruments can be used. An irrigation-suction cannula and bipolar cautery are pivotal for the procedure. Isobaric laparoscopic myomectomy has a few advantages. First, it avoids building pneumoperitoneum, thereby avoiding the side effects and potential risks of $\mathrm{CO}_{2}$. Second, because the peritoneal cavity does not need to maintain pressure-tight, conventional, long laparotomy instruments, including knives, scissors, and tissue clamps, needle holders can be used. This facilitates several steps of the procedure, including uterine repair. Third, operative costs and operative times are reduced. Furthermore, this method is more easily learned by surgeons who are experienced with laparotomy.

\section{Trocar placement}

Correcting the trocar placement is a crucial factor that influences the surgical procedure. Traditionally, surgeons place three portals during surgery. However, with developments in the surgery technique and the higher demand for the operation, more portal sites are being used, and each has its own merits.

\section{Conventional portal sites and the Lee-Huang point}

The conventional use of three portal sites is good for medium masses. Video-assisted laparoscopy was performed through the umbilicus using a $5-\mathrm{mm}$ or $10-\mathrm{mm}$ principal trocar. Two ancillary cannulas are placed with the help of video-assisted laparoscopy: one 5-mm trocar in the right lower quadrant beside the inferior epigastric arteries and the other 5-mm trocar in the left lower quadrant [1]. However, with the demand for larger fibroids, Lee et al. introduced new portal sites called the Lee-Huang point. The principal cannula is placed at the midpoint between the umbilicus and the diploid process. Another two 5-mm puncture sites are made at the intersection of the bilateral paramedian line and the level of umbilicus. If three portal sites are not enough, then other trocars can be selected just above the pubic hairline and at the level of the paramedian line.

This type of placement has many advantages. First, the placement of the first trocar (the Lee-Huang point) is much safer for patients with a history of pelvic surgery, potential malignancies, and large pelvic masses. This placement of the cannulas avoids the major bloods vessels and nerves. Second, the incision through the linea alba accesses the abdominal cavity better, provides a better visual field, and increases the accuracy when resecting large myomas. Therefore, using the Lee-Huang point may be the best choice for large masses.

\section{Laparoendoscopic single-site myomectomy}

Some research has demonstrated that laparoendoscopic single-site myomectomy (LESS-M) is suitable for patients 
with fewer than five myomas and offers outcomes akin to those of conventional laparoscopic myomectomy after surgeons master the technique [20]. However, LESS has not been widely popularized because of its difficult technique that involves limited motion or clashing between instruments. Another challenge for LESS-M is that after the large tissue specimens are removed through the umbilical incision, the uterine walls need multiple sutures; therefore, repair of the uterine wall injuries may be difficult for surgeons with limited experience. Furthermore, difficulties in maintaining adequate tension of the suture line and trying to accurately create a knot increase the operative time and intraoperative blood loss. Only a wound retractor and a surgical glove can fit in a single port entry. A vertical incision of 15-20 mm was made through the umbilicus; then, two 5-mm cannulas and one 12-mm cannula were inserted with the first, third, and fifth fingers of the surgical glove and fixed with silk ligatures. Next, the glove was fixed at the outer ring of the wound retractors. After repair of the myometrium, surgeons use an electromechanical morcellator, which is usually $15 \mathrm{~mm}$, to enucleate myomas, and that device can be placed with one free gloved finger [20].

Although difficult, LESS-M has some advantage. Obviously, its cosmetic benefits are greater than those of other surgery types. Some studies showed that LESS can reduce postoperative pain more than conventional laparoscopic surgery [21]. For instance, the lower abdomen large trocar insertions of conventional laparoscopic surgery may increase the risk of trocar-related sequela, such as incisional hernias [22].

\section{Choi's four-trocar method}

In 2006, Choi et al. [23] introduced the four-trocar method, which provides a better operation field for the large uterus. The method uses four trocars for patients whose uterus is same size as it at approximately 16 to 18 weeks of pregnancy. With the guidance of a central 5-mm telescope, the placement of the first trocar is usually the supraumbilical region. Another two 5-mm ancillary trocars are placed lateral to the superior and inferior epigastric vessels in the left and right upper quadrants. The level of the upward shift can be modulated by the uterine size. The fourth trocar is placed $2 \mathrm{~cm}$ above the symphysis pubis so that the four 5-mm trocar points can form a V-shape.

This technique has many benefits. First, surgeons can obtain a larger surgical field to exclude the myoma. Second, all the trocars are situated in the "safe zone" to avoid potential injury to the surrounding nerves and vessels, especially the ilioinguinal or iliohypogastric nerves and the inferior epigastric arteries. Third, the risk of intestinal herniation and the scar size are reduced because only 5-mm trocar ports and one 12-mm cannula are used for the myoma screw or morcellator.
However, some believe that because the uterus is large, the placement of the primary trocar in the umbilical region makes the working distance shorter and the operation field smaller. It may be difficult for surgeons to view the whole pelvis, large uterus, and tumors. Therefore, the Lee-Huang point, may be a better option.

\section{Two-port total laparoscopic myomectomy}

Two-port total laparoscopic myomectomy (TTLM) was performed using only umbilicus and left inguinal ports. An Olympus 5-mm flexible scope was used to visualize the surgical field, regardless of the insertion angle, and a 12$\mathrm{mm}$ trocar was placed at the umbilical incision. However, for the patients with a history of open abdominal surgery, the ninth intercostal approach is necessary to confirm the absence of any adhesion of the abdominal organs and the umbilicus [24].

The technique has the same technical difficulty as the conventional technique, and it allows exact suturing of the myometrium ( 2 to 4 layers) assuming the myomas being removed are within the indicated limits. Furthermore, the two-port technique provides a much higher degree of freedom when handling forceps and adjusting the surgical field of view than single-port myomectomy.

\section{Is morcellation an Achilles heel?}

Electrical morcellators have been used during laparoscopy; however, they are associated with complications, including major vascular, bowel, ureteric, kidney, and diaphragmatic injuries [25]. These complications are rare. Other less immediately obvious and long-term complications associated with electrical morcellators are more common. A morcellator is in fact a cylinder device with a cutting tip that can rotate rapidly. It cuts the tumor into strips and then collects those strips in its hollow cylindrical body. However, some of the strips will fall out of the device and must be collected and removed individually. However, some small particles or cells in microscopic quantities can spin off and cannot be removed. These may develop into disseminated peritoneal leiomyomatosis in the future. Characteristics of this type of disease include multiple smooth muscle nodules that can develop sub-peritoneally and can be found in any part of the abdominal cavity; although morbidity is rare and most cases remain benign, some may progress to cancer [26]. It may be not worth the risk and lead to a poor prognosis for patients.

An animal model that was established to study the mechanism of the parasite myoma found that estrogen has an important role. Implanted myomas possess more estrogen receptor (ER) and more progesterone receptors (PR), and they have more angiogenesis and proliferative properties compared with non-implanted myomas. Estro- 
gen depletion will significantly decrease laparoscopically induced parasite myoma implantation. The implantation, angiogenesis, and proliferation of parasite myomas may be associated with serum E2 levels. Sex steroid hormone modulators and aromatase inhibitors (AI) may also decrease implantation, angiogenesis, and proliferation. These data revealed that angiogenesis and implantation induced by estrogen have an important role in the development of parasite myomas, and that hormonal modulation with $\mathrm{Al}$ could potentially prevent laparoscopically induced parasite myomas [27].

In clinical practice, surgeons have created many measures to prevent parasite myomas. A thorough pelvic lavage is required during surgery because it may reduce the cellular load. Some surgeons use a plastic bag to collect morcellation materials and then dilate the navel scar to $3-4 \mathrm{~cm}$ so the bag can be removed through the navel [28]. Some experts think that avoiding the use of an electronic morcellator can achieve satisfactory tumor reduction during laparoscopic surgery. Reich et al. introduced a method using conventional surgical tools, such as a scalpel, that can morcellate the largest tumors. This method only requires a small incision in the abdominal wall to insert the device [29]. Another specifically designed morcellator knife has been shown to decrease the loss of the tissue during surgery [30]. Many types of new morcellation tools are in varying degrees of development. For example, devices that can be used outside the abdomen, such as with the vaginal approach, are being developed.

\section{Laparoscopic radiofrequency volumetric thermal ablation (RFVTA)}

Laparoscopic radiofrequency volumetric thermal ablation (RFVTA) based on primary liver ablation was developed by Lee in 2002 [31]. The equipment used for RFVTA treatment include a monopolar radiofrequency generator, a handpiece with an electrode tip, two electrode pads, extension cables, an activating foot pedal, and other equipment. Current is delivered to the small electrode tip to ablate the fibroid tissue, which is removed via two large dispersive electrode pads that are usually placed on the patient's thighs. When the electrical current decreases, it results in the oscillation of intracellular ions, thus generating resistive or frictional heating. During the procedure, the heat decreases rapidly with the increasing distance from the electrode. The current continues to flow from the handpiece to the electrode pads; at the same time, the myomas undergo ablation and become coagulative and necrosis. Finally, they are reabsorbed by the surrounding tissues.

As a minimally invasive and uterine-sparing procedure, RFVTA has many advantages that cannot be duplicated. More fibroids can be detected using laparoscopic ultra- sound intraoperatively than with either transvaginal ultrasound or contrast-enhanced magnetic resonance imaging (MRI) [32]; therefore, it is suitable for more cases such as large fibroids, multiple fibroids, and deep intramural fibroids [33], but not for type 0 (pedunculated) intracavitary fibroids, which are best suited to undergo hysteroscopic resection. Because RFVTA uses flowing current to coagulate fibroids, there is less blood loss and no laparoscopic uterine suturing, which is difficult for many surgeons. Surgeons only need to use intracutaneous sutures to close the port sites; therefore, patients often have minimal injury and leave the hospital on the same day as the surgery [34]. Furthermore, RFVTA provides significant reductions in uterine size, significant reductions in or elimination of myoma symptoms, and significant improvements in quality of life [35]. The reproductive outcomes of RFVTA are also positive. Berman et al. analyzed the pregnancy outcomes of six women who conceived 3.5 to 15 months after the treatment of one to seven myomas that were between 1.0 and $7.6 \mathrm{~cm}$ at the greatest diameter and were of multiple types. Five of the women delivered full-term, healthy newborns and one had a spontaneous abortion during the first trimester. Because RFVTA is a new minimally invasive alternative for uterine myoma, further investigations are needed to determine whether it is appropriate treatment for women who desire future fertility.

Some research has focused on comparing the differences between RFVTA and laparoscopic myomectomy, which is a classic minimally invasive option. It was found that RFVTA results in shorter hospitalizations, less blood loss, and a greater percentage of fibroids treated/excised than laparoscopic myomectomy [36]. Although laparoscopic myomectomy may cause a more significant improvement in health-related quality of life and decreased symptom severity scores, laparoscopic myomectomy and RFVTA may have equivalent safety and patient-reported efficacy [37].

\section{Laparoscopic myolysis}

The Nd YAG laser was first considered an alternative to laparoscopic myomectomy in 1989 because for some cases, such as those involving multiple intramural myomas, laparoscopic myomectomy is too difficult or too time-consuming. Indications for laparoscopic myolysis include pelvic pain caused by myoma, compression symptoms, or global uterine volumes equivalent to those between 9 and 12 weeks of pregnancy [38]. The Nd YAG laser technique involves bringing the tissue fiber into the center of the myoma so that the fibroids can be coagulated. The diameter of fibroids suitable for myolysis usually range from 3 to $8 \mathrm{~cm}$. The mean decrease in the myoma diameter after myolysis was $41 \%$ after 6 months. However, laparoscopic myolysis also has some complications, such as severe pain caused by coagulation of 
the myomas, the risk of uterine rupture during pregnancy, uterine abscesses, and pelvic adhesions [39].

\section{Uterine artery embolization}

Uterine artery embolization (UAE) was first created in 1995 to treat typical uterine fibroids [40]. It may be an alternative to traditional treatment, especially for women with multiple fibroids, very large fibroids, restricted operability, or a history of multiple operative procedures in the abdomen [41]. UAE has some contraindications, including viable pregnancy, active infection, and suspected uterine, cervical, or adnexal malignancy [42]. However, UAE has many advantages, such as less blood loss, shorter operative times, and shorter hospital stays [43]. The technique also has some limitations that prevent its widespread application, such as postembolization syndrome, which includes complete amenorrhea [44], subclinical damage of the ovarian function (especially in women older than 45 years) [45], and the potential risk of reintervention or subsequent hysterectomy [46]. Furthermore, the time required for the procedure and the radiation exposure also prevent the application of UAE [47]. UAE is not suitable for women who want to preserve their fertility.

Regarding the prognosis for UAE, Davis et al discussed the reintervention rates among myomectomy, UAE, and endometrial ablation (EA). They found that 5 years after surgery, the reintervention rate for UAE was lower than that for EA but higher than that for myomectomy. Prior anemia, bleeding, pelvic inflammatory disease, and pelvic pain might increase the risk of reintervention [48]. Karlsen et al reported that UAE may lead to lower pregnancy rates and higher miscarriage rates than myomectomy [49]. Therefore, although UAE is a safe method with many benefits, patient selection and counseling are important.

\section{Laparoscopic uterine artery ligation (LUAL)}

The principle of laparoscopic uterine artery ligation (LUAL) is similar to that of UAE. Both the right and left uterine arteries need to be ligated by the hemoclips, followed by bipolar coagulation. LUAL can overcome the complications of UAE. However, more research and comparisons, such as with hysterectomy and LAVH, are needed.

\section{Magnetic resonance-guided ultrasound}

Magnetic resonance (MR)-guided ultrasound is not widely used because of the costly equipment required. Furthermore, few long-term randomized studies have been performed. MR-guided ultrasound is composed of two parts. MRI is used for treatment planning and synchronous treatment monitoring. Ultrasound is used for necrotizing tissue, which involves heating the fibroids to 60 to $80^{\circ} \mathrm{C}$ and leads to a reduction in fibroid size. Absolute contraindications for the technique are ongoing pregnancy and all contraindications for MRI. However, large fibroid size itself is not a contraindication. Furthermore, some complications may occur, such as skin burns, postoperative pain, nausea, and allergic reactions [50]. Therefore, more clinical research is necessary to verify the value and safety of this technique.

\section{CONCLUSIONS}

Because uterine fibroids are one of the most common female pelvic tumors, the development of operative methods of their treatment will continue. Furthermore, because of the rapid progression of operative techniques and surgical skills, more surgeons and patients prefer minimally invasive methods to treat uterine fibroids. At first, minimally invasive surgery could only be used to treat small subserous fibroids. Now, the indications for minimally invasive surgery are becoming broader, case reports are increasing, fibroid sizes are becoming larger, the number of fibroids is increasing, and fibroid locations are becoming more remote. However, are these surgeries obligatory? Minimally invasive surgery has more shortcomings than traditional laparotomy, such as the longer operative times, the lower suturing accuracy, and the greater potential for pelvic adhesions. More research is necessary to evaluate the indications for minimally invasive surgery to determine whether the outcomes are worth the risks.

Currently, electrical morcellation is a crucial component of minimally invasive surgery because it can help decrease invasiveness and hasten the surgery. However, electrical morcellation has some side effects, such as parasite myomas. Parasite myomas can develop in any part of the abdominal cavity, and multiple smooth muscle nodules can develop sub-peritoneally. Although its morbidity is rare and most cases are benign, some may progress to cancer. Many solutions have been suggested for this complication, but none has been promoted worldwide.

The main purpose of minimally invasive surgery is to decrease injury during surgery and shorten the recovery time. Achieving this goal cannot be accomplished with only minimally invasive surgery. According to enhanced recovery after surgery (ERAS), many measures are necessary, from preparation before surgery to recovery after surgery, such as bowel preparation and adaptive training before surgery, fluid management and temperature control during surgery, and analgesia and retention of the drainage tube after surgery, among others. Minimally invasive surgery should be a component of ERAS so that minimal invasiveness and faster recovery can be realized. 


\section{Funding}

This study was supported by Program for Shenyang Science Foundation(No.19-112-4-020) and Natural Science Foundation of Liaoning Province (No.JC2019012).

\section{REFERENCES}

1. Lee $\mathrm{CL}$, Wang CJ. Laparoscopic Myomectomy. Taiwanese Journal of Obstetrics and Gynecology. 2009; 48(4): 335-341, doi: 10.1016/s10284559(09)60321-1.

2. Huang HY, Liu YC, Li YC, et al. Comparison of three different hemostatic devices in laparoscopic myomectomy. J Chin Med Assoc. 2018; 81(2): 178-182, doi: 10.1016/j.jcma.2017.04.012, indexed in Pubmed: 29129517.

3. Bhave Chittawar P, Franik S, Pouwer AW, et al. Minimally invasive surgical techniques versus open myomectomy for uterine fibroids. Cochrane Database Syst Rev. 2014(10): CD004638, doi: 10.1002/14651858.CD004638. pub3, indexed in Pubmed: 25331441.

4. Bojahr B, De Wilde RL, Tchartchian G. Malignancy rate of 10,731 uteri morcellated during laparoscopic supracervical hysterectomy (LASH). Arch Gynecol Obstet. 2015; 292(3): 665-672, doi: 10.1007/s00404-0153696-z, indexed in Pubmed: 25820974.

5. Macciò A, Kotsonis $P$, Lavra F, et al. Laparoscopic removal of a very large uterus weighting $5320 \mathrm{~g}$ is feasible and safe: a case report. BMC Surg. 2017; 17(1): 50, doi: 10.1186/s12893-017-0248-4, indexed in Pubmed: 28472966.

6. Cianci S, Gueli Alletti S, Rumolo V, et al. Total laparoscopic hysterectomy for enlarged uteri: factors associated with the rate of conversion to open surgery. J Obstet Gynaecol. 2019; 39(6): 805-810, doi: 10.1080/01443615.2019.1575342, indexed in Pubmed: 31001998.

7. Shiota $M$, Kotani $Y$, Umemoto $M$, et al. Indication for laparoscopically assisted vaginal hysterectomy. JSLS. 2011; 15(3): 343-345, doi: 10.4293 /108680811X13125733357151, indexed in Pubmed: 21985721.

8. McGurk L, Oliver R, Odejinmi F. Laparoscopic supracervical hysterectomy for the larger uterus ( $>500 \mathrm{~g}$ ): a case series and literature review. Arch Gynecol Obstet. 2017; 295(2):397-405, doi: 10.1007/s00404-016-4237-0, indexed in Pubmed: 27848016.

9. Yuan $\mathrm{H}$, Wang $\mathrm{C}$, Wang $\mathrm{D}$, et al. Comparing the effect of laparoscopic supracervical and total hysterectomy for uterine fibroids on ovarian reserve by assessing serum anti-mullerian hormone levels: a prospective cohort study. J Minim Invasive Gynecol. 2015; 22(4): 637-641, doi: 10.1016/j. jmig.2015.01.025, indexed in Pubmed: 25653041.

10. Wallwiener M, Taran FA, Rothmund R, et al. Laparoscopic supracervical hysterectomy (LSH) versus total laparoscopic hysterectomy (TLH): an implementation study in 1,952 patients with an analysis of risk factors for conversion to laparotomy and complications, and of procedure-specific re-operations. Arch Gynecol Obstet. 2013; 288(6): 1329-1339, doi: 10.1007/s00404-013-2921-x, indexed in Pubmed: 23775263.

11. Brucker SY, Taran FA, Bogdanyova S, et al. Patient-reported quality-of-life and sexual-function outcomes after laparoscopic supracervical hysterectomy (LSH) versus total laparoscopic hysterectomy (TLH): a prospective, questionnaire-based follow-up study in 915 patients. Arch Gynecol Obstet. 2014; 290(6): 1141-1149, doi: 10.1007/s00404-014-3318-1, indexed in Pubmed: 24973868.

12. Deslyn TG, et al. Hobson - Anthony N. Comparative analysis of different laparoscopic hysterectomy procedures. Arch Gynecol Obstet. 2012; 285: 1353-1361.

13. Borgfeldt $C$, Andolf $E$. Transvaginal ultrasonographic findings in the uterus and the endometrium: low prevalence of leiomyoma in a random sample of women age 25-40 years. Acta Obstet Gynecol Scand. 2000; 79(3): 202-207, indexed in Pubmed: 10716301.

14. Hu Y, Yu L, Xia F, et al. Effect of laparoscopic myomectomy on serum levels of IL-6 and TAC, and ovarian function. Exp Ther Med. 2019; 18(5): 3588-3594, doi: 10.3892/etm.2019.7941, indexed in Pubmed: 31602235.

15. Kotani Y, Tobiume T, Fujishima R, et al. Recurrence of uterine myoma after myomectomy: Open myomectomy versus laparoscopic myomectomy. J Obstet Gynaecol Res. 2018; 44(2): 298-302, doi: 10.1111/jog.13519, indexed in Pubmed: 29227004.

16. Stoica RA, Bistriceanu I, Sima R. Laparoscopic myomectomy . Journal of Medicine and Life. 2014; 7(4): 522-524.

17. Ksiezakowska-Lakoma K, Zyla K, Wilczynski J. Removal of uterine fibroids by mini-laparotomy technique in women who wish to preserve their uterus and fertility. 2015; 10(4): 561-566.
18. Yuen $L T$, Hsu LJ, Lee CL, et al. A modified suture technique for laparoscopic myomectomy. J Minim Invasive Gynecol. 2007; 14(3): 318-323, doi: 10.1016/j.jmig.2006.11.008, indexed in Pubmed: 17478362.

19. Sesti F, Pietropolli A, Sesti FF, et al. Uterine myomectomy: role of gasless laparoscopy in comparison with other minimally invasive approaches. Minim Invasive Ther Allied Technol. 2013; 22(1): 1-8, doi: 10.3109/13645706.2012.680889, indexed in Pubmed: 22554032.

20. Kim SKi, Lee JiH, Lee JR, et al. Laparoendoscopic single-site myomectomy versus conventional laparoscopic myomectomy: a comparison of surgical outcomes. J Minim Invasive Gynecol. 2014; 21(5): 775-781, doi: 10.1016/j.jmig.2014.03.002, indexed in Pubmed: 24632396.

21. Kim TJ, LeeYY, Cha HH, et al. Single-port-access laparoscopic-assisted vaginal hysterectomy versus conventional laparoscopic-assisted vaginal hysterectomy: a comparison of perioperative outcomes. Surg Endosc. 2010; 24(9): 2248-2252, doi: 10.1007/s00464-010-0944-y, indexed in Pubmed: 20177921.

22. Kim SKi, Lee JiH, Lee JR, et al. Laparoendoscopic single-site myomectomy versus conventional laparoscopic myomectomy: a comparison of surgical outcomes. J Minim Invasive Gynecol. 2014; 21 (5): 775-781, doi: 10.1016/j.jmig.2014.03.002, indexed in Pubmed: 24632396.

23. Choi JS, Kyung YS, Kim KH, et al. The four-trocar method for performing laparoscopically-assisted vaginal hysterectomy on large uteri. J Minim Invasive Gynecol. 2006; 13(4): 276-280, doi: 10.1016/j.jmig.2006.04.004, indexed in Pubmed: 16825066.

24. Kikuchi I, Kumakiri J, Matsuoka S, et al. Learning curve of minimally invasive two-port laparoscopic myomectomy. JSLS. 2012; 16(1): 112-118, doi: 10.4293/108680812X13291597716267, indexed in Pubmed: 22906339

25. Milad MP, Milad EA. Laparoscopic morcellator-related complications. J Minim Invasive Gynecol. 2014; 21(3): 486-491, doi: 10.1016/j. jmig.2013.12.003, indexed in Pubmed: 24333632.

26. Garry R. Laparoscopic morcellation: an acceptable risk or an Achilles heel? BJOG. 2015; 122(4): 458-460, doi: 10.1111/1471-0528.13045, indexed in Pubmed: 25236787.

27. Huang $\mathrm{BS}$, Yang $\mathrm{MH}$, Wang $\mathrm{PH}$, et al. Oestrogen-induced angiogenesis and implantation contribute to the development of parasitic myomas after laparoscopic morcellation. Reprod Biol Endocrinol. 2016; 14(1): 64, doi: 10.1186/s12958-016-0200-y, indexed in Pubmed: 27716434.

28. Chikazawa K, Netsu S, Konno R. Myoma morcellation through the navel. Taiwan J Obstet Gynecol. 2015; 54(1): 106, doi: 10.1016/j. tjog.2014.11.020, indexed in Pubmed: 25675936.

29. Reich H. Laparoscopic MYOMECTOMY. In: Hutchins FL, Greenberg MD, editors. . Obstetrics and Gynecology Clinics of North America: Uterine Fibroids. 1995; 22: 756-780.

30. De Grandi $P$, Chardonnens $E$, Gerber $S$. The morcellator knife: a new laparoscopic instrument for supracervical hysterectomy and morcellation. Obstet Gynecol. 2000; 95(5): 777-778, doi: 10.1016/s00297844(99)00625-0, indexed in Pubmed: 10841695.

31. LEE B. Radiofrequency ablation of uterine leiomyomata: a new minimally invasive hysterectomy alternative. Obstetrics \& Gynecology. 2002; 99(4): S9, doi: 10.1016/s0029-7844(02)01682-4.

32. Levine DJ, Berman JM, Harris $M$, et al. Sensitivity of myoma imaging using laparoscopic ultrasound compared with magnetic resonance imaging and transvaginal ultrasound. J Minim Invasive Gynecol. 2013; 20(6): 770-774, doi: 10.1016/j.jmig.2013.04.015, indexed in Pubmed: 24021910.

33. Brucker SY, Hahn M, Kraemer D, et al. Laparoscopic radiofrequency volumetric thermal ablation of fibroids versus laparoscopic myomectomy. Int J Gynaecol Obstet. 2014; 125(3): 261-265, doi: 10.1016/j. ijgo.2013.11.012, indexed in Pubmed: 24698202.

34. Donnez J, Mathieu PE, Bassil S, et al. Laparoscopic myomectomy today. Fibroids: management and treatment: the state of the art. Hum Reprod. 1996; 11(9): 1837-1840, doi: 10.1093/oxfordjournals.humrep.a019502, indexed in Pubmed: 8921049.

35. Galen DI, Pemueller RR, Leal JG, et al. Laparoscopic radiofrequency fibroid ablation: phase II and phase III results. JSLS. 2014; 18(2): 182-190, doi: 10.4293/108680813X13693422518353, indexed in Pubmed: 24960480.

36. Brucker SY, Hahn M, Kraemer D, et al. Laparoscopic radiofrequency volumetric thermal ablation of fibroids versus laparoscopic myomectomy. Int J Gynaecol Obstet. 2014; 125(3): 261-265, doi: 10.1016/j. ijgo.2013.11.012, indexed in Pubmed: 24698202.

37. Hahn M, Brucker S, Kraemer D, et al. Radiofrequency Volumetric Thermal Ablation of Fibroids and Laparoscopic Myomectomy: Long-Term Follow-up From a Randomized Trial. Geburtshilfe Frauenheilkd. 2015; 75(5): 442-449, doi: 10.1055/s-0035-1545931, indexed in Pubmed: 26097247. 
38. Quinn SD, Gedroyc WM. Thermal ablative treatment of uterine fibroids. Int J Hyperthermia. 2015; 31(3): 272-279, doi: 10.3109/02656736.2015.1010608, indexed in Pubmed: 25815582.

39. Hai N, Ding X. Intrauterine adhesion after transvaginal ultrasound-guided radiofrequency myolysis. J Obstet Gynaecol Res. 2015; 41(11): 1851-1854, doi: 10.1111/jog.12796, indexed in Pubmed: 26311404.

40. Ravina JH, Herbreteau D, Ciraru-Vigneron N, et al. Arterial embolisation to treat uterine myomata. Lancet. 1995; 346(8976): 671-672, doi: 10.1016/s0140-6736(95)92282-2, indexed in Pubmed: 7544859.

41. Mavrelos D, Ben-Nagi J, Davies A, et al. The value of pre-operative treatment with $\mathrm{GnRH}$ analogues in women with submucous fibroids: a double-blind, placebo-controlled randomized trial. Hum Reprod. 2010; 25(9): 2264-2269, doi: 10.1093/humrep/deq188, indexed in Pubmed: 20663795.

42. Kohi MP, Spies JB. Updates on Uterine Artery Embolization. Semin Intervent Radiol. 2018; 35(1): 48-55, doi: 10.1055/s-0038-1636521, indexed in Pubmed: 29628616.

43. Boosz A, Reimer $P$, Matzko $M$, et al. The Conservative and Interventional Treatment of Fibroids. Deutsches Aerzteblatt Online. 2014, doi: 10.3238/arztebl.2014.0877.

44. Toor SS, Jaberi A, Macdonald DB, et al. Complication rates and effectiveness of uterine artery embolization in the treatment of symptomatic leiomyomas: a systematic review and meta-analysis. AJR Am J Roentgenol. 2012; 199(5): 1153-1163, doi: 10.2214/AJR.11.8362, indexed in Pubmed: 23096193.

45. Sentilhes L, Vayssière C, Beucher G, et al. Delivery for women with a previous cesarean: guidelines for clinical practice from the French College of
Gynecologists and Obstetricians (CNGOF). Eur J Obstet Gynecol Reprod Biol. 2013; 170(1): 25-32, doi: 10.1016/j.ejogrb.2013.05.015, indexed in Pubmed: 23810846.

46. van der Kooij SM, Bipat S, et al. Uterine artery embo- lization versus surgery in the treatment of symptomatic fibroids: a systematic review and metaanalysis. Am J Obstet Gynecol . 2011; 205: 317-318.

47. Kim HS, Kim JW, Kim MK, et al. A randomized prospective trial of the postoperative quality of life between laparoscopic uterine artery ligation and laparoscopy-assisted vaginal hysterectomy for the treatment of symptomatic uterine fibroids: clinical trial design. Trials. 2009; 10: 8 , doi: 10.1186/1745-6215-10-8, indexed in Pubmed: 19178748.

48. Davis MR, Soliman AM, Castelli-Haley J, et al. Reintervention Rates After Myomectomy, Endometrial Ablation, and Uterine Artery Embolization for Patients with Uterine Fibroids. J Womens Health (Larchmt). 2018; 27(10): 1204-1214, doi: 10.1089/jwh.2017.6752, indexed in Pubmed: 30085898.

49. Karlsen K, Hrobjartsson A, Korsholm M, et al. Fertility after uterine artery embolization of fibroids: a systematic review. Arch Gyneco Obstet. 2018; 297(1): 13-25, doi: 10.1007/s00404-017-4566-7, indexed in Pubmed: 29052017.

50. Trumm CG, Stahl R, Clevert DA, et al. Magnetic resonance imaging-guided focused ultrasound treatment of symptomatic uterine fibroids: impact of technology advancement on ablation volumes in 115 patients. Invest Radiol. 2013; 48(6): 359-365, doi: 10.1097/RLI.0b013e3182806904, indexed in Pubmed: 233853 